\title{
Aarno Sífilis gestacional e congênita e sua relação com a cobertura da Estratégia Saúde da Família, Goiás, 2007-2014: um estudo ecológico*
}

doi: 10.5123/S1679-49742018000400008

\author{
Syphilis in pregnancy and congenital syphilis and their relationship with Family Health \\ Strategy coverage, Goiás, Brazil, 2007-2014: an ecological study
}

\section{Sífilis gestacional y congénita y su relación con la cobertura de la Estrategia Salud de la Familia, Goiás, Brasil, 2007-2014: un estudio ecológico}

\author{
Patrícia Silva Nunes ${ }^{1}$ - (1) orcid.org/0000-0003-4163-9516 \\ Ana Laura de Sene Amâncio Zara' \\ Déborah Ferreira Noronha de Castro Rocha ${ }^{2}$ \\ Tamíris Augusto Marinho ${ }^{3}$ \\ Polyana Maria Pimenta Mandacarú \\ Marília Dalva Turchi' \\ 'Universidade Federal de Goiás, Instituto de Patologia Tropical e Saúde Pública, Goiânia, GO, Brasil \\ ${ }^{2}$ Secretaria de Estado da Saúde de Goiás, Coordenação Estadual de DST/Aids, Goiânia, GO, Brasil \\ ${ }^{3}$ Instituto Federal de Educação, Ciência e Tecnologia de Goiás, Núcleo de Estudos e Pesquisas em Promoção da Saúde, Goiânia, GO, Brasil
}

\section{Resumo}

Objetivo: analisar a incidência de sífilis em gestante (SG) e sífilis congênita (SC) e a correlação desses indicadores com a cobertura da Estratégia Saúde da Família (ESF) em Goiás, Brasil, de 2007 a 2014. Métodos: estudo ecológico utilizando dados do Sistema de Informação de Agravos de Notificação (Sinan) e do Departamento de Atenção Básica (DAB)/Ministério da Saúde; utilizou-se a correlação de Spearman para avaliar a relação entre cobertura da ESF e a incidência de sífilis. Resultados: a incidência de SG passou de 2,8 para 9,5/mil nascidos vivos, e a de $\mathrm{SC}$, de 0,3 para 2,5/mil nascidos vivos $(\mathrm{p}<0,05)$, no período 2007-2014; houve aumento significativo de casos de SC nos municípios que apresentaram percentuais de cobertura da ESF inferiores a 75\% ( $<<0,001)$. Conclusão: o aumento concomitante da incidência de SG e de SC sugere falhas na prevenção da transmissão vertical da sífilis, sobretudo nas regiões com menor cobertura da ESF.

Palavras-chave: Sífilis; Sífilis Congênita; Gestantes; Estratégia Saúde da Família; Estudos Ecológicos.

\footnotetext{
*Artigo elaborado a partir da dissertação de mestrado da autora principal, Patrícia Silva Nunes, intitulada 'A síflis congênita no estado de Goiás, 2007 a 2014', defendida junto ao Programa de Pós-Graduação em Saúde Coletiva da Universidade Federal de Goiás em 11 de dezembro de 2015.
}

Endereço para correspondência:

Patrícia Silva Nunes - Rua 226, c/ 227, Residencial Bucareste, Torre F, apto. 301, Setor Leste Universitário, Goiânia, GO, Brasil. CEP: 74610-130

E-mail:patnunesufg@gmail.com 


\section{Introdução}

A Organização Mundial da Saúde (OMS) estima que, anualmente, ocorra mais de 1 milhão de casos novos de gestantes infectadas pelo Treponema pallidum em todo o mundo, com risco de desfechos adversos em 65\% dessas gestações. ${ }^{1}$ Frente a esse cenário, iniciativas mundiais foram desencadeadas para eliminação da transmissão vertical da sífilis até 2015 , visando alcançar incidências inferiores a 0,5 caso por cada mil nascidos vivos. Para alcançar essa meta, foi estipulado que pelo menos $90 \%$ das gestantes soropositivas para sífilis recebessem tratamento adequado., ${ }^{1,2}$

Sabe-se que gestantes diagnosticadas e tratadas precocemente apresentam redução do risco de transmissão vertical da sífilis e menor chance de apresentarem desfechos desfavoráveis ao concepto, se comparadas àquelas com intervenção medicamentosa tardia. ${ }^{3} 0$ cuidado pré-natal inadequado, por sua vez, é um fator crucial para 0 aumento de casos de sífilis congênita (SC), haja vista a oportunidade de diagnóstico e tratamento apresentar-se, em sua maioria, durante esse período. ${ }^{4,5}$

A Estratégia Saúde da Família (ESF), proposta como modelo de atenção à saúde pela Política Nacional de Atenção Básica, tem contribuído para a ampliação da oferta de serviços de saúde no Brasil. Todavia, a ESF apresenta desafios substanciais quanto ao acesso integral e oportuno desses serviços pela população. ${ }^{6}$

\section{Gestantes diagnosticadas e tratadas precocemente apresentam redução do risco de transmissão vertical da sífilis e menor chance de apresentarem desfechos desfavoráveis ao concepto.}

0 pré-natal é um importante componente do cuidado oferecido pelas equipes de saúde da família e constitui um momento primordial para o manejo adequado de infecções passíveis de transmissão vertical, como a sífilis, doença capaz de elevar o risco de perda fetal em até $21 \%$ em gestantes infectadas, quando comparadas àquelas sem a infecção. ${ }^{7}$

No âmbito do Sistema Único de Saúde (SUS), em 2011 foi criada a Rede Cegonha, estabelecendo-se diretrizes para um atendimento materno-infantil de qualidade. A redução da SC foi proposta como uma estratégia essencial do componente pré-natal.
Foi preconizada a ampliação da testagem rápida para sífilis no primeiro e no terceiro trimestres de gestação, com o objetivo de viabilizar o diagnóstico e $o$ tratamento em tempos oportunos. ${ }^{8}$ Pressupõe-se que a ampliação do acesso da gestante à rede de serviços de pré-natal no Brasil, preferencialmente na atenção primária à saúde prestada pela $\mathrm{ESF}^{8}$ seja um importante aliado na redução dos agravos à saúde da mãe e do concepto, entre eles a transmissão vertical da sífilis.

0 presente estudo teve por objetivo analisar a incidência de sífilis em gestante (SG) e de sífilis congênita (SC) e a correlação desses indicadores com a cobertura da ESF no estado de Goiás, entre 2007 e 2014.

\section{Métodos}

Foi realizado um estudo ecológico dos casos registrados de SC e SG no estado de Goiás, além da cobertura da ESF nos municípios goianos notificantes, entre os anos de 2007 e 2014.

A população do estado de Goiás em 2015 estava estimada em 6.778 .772 habitantes, distribuídos entre 246 municípios, estes agrupados em cinco denominadas Macrorregiões de Saúde: Nordeste, Centro-Oeste, Centro-Norte, Sudeste e Sudoeste. ${ }^{9}$

A população-alvo de estudo foi composta por todos os casos de SC e SG notificados em Goiás, por município e macrorregião de saúde, durante o período em estudo. A ESF foi considerada como proxy de acesso aos serviços de saúde, incluindo a assistência pré-natal.

As variáveis dependentes foram o coeficiente de incidência de SC e o coeficiente de detecção de SG, por mil nascidos vivos. As variáveis independentes incluíram a cobertura da ESF nos municípios que notificaram casos de SC e SG, agrupados em quatro intervalos segundo o percentual de cobertura da Saúde da Família - $1^{\circ}$ intervalo, $\leq 25 \% ; 2^{\circ}$ intervalo, $>25 \%$ e $<50 \% ; 3^{\circ}$ intervalo, $\geq 50 \%$ e $<75 \%$; $4^{\circ}$ intervalo, $\geq 75 \%$ ) - e macrorregiões de saúde do estado (Centro-Oeste, Nordeste, Centro-Norte, Sudoeste e Centro-Sudeste).

Os dados foram extraídos dos seguintes sistemas de informações em saúde em Goiás: Sistema de Informação dos Agravos de Notificação (Sinan); Sistema de Informações sobre Nascidos Vivos (Sinasc); e Sistema de Informação da Atenção Básica (Siab), via Departamento de Atenção Básica (DAB) da Secretaria de Atenção à Saúde do Ministério da Saúde. 
Os dados referentes ao percentual de cobertura da ESF por municípios foram importados da página eletrônica do DAB, considerando-se o mês de junho de cada ano.

Para o cálculo dos coeficientes de incidência de SC, utilizou-se o número de casos notificados por ano de diagnóstico e local de residência, dividido pelo número de nascidos vivos no mesmo local e ano, multiplicado pela constante 1.000. Os coeficientes de detecção de SG foram obtidos da razão entre o número de gestantes notificadas com sífilis e o número de nascidos vivos no mesmo ano e local, multiplicada pela constante de 1.000 nascidos vivos.

Para análise da correlação entre a cobertura da ESF e a incidência de sífilis (SG e SC), foram considerados os municípios que notificaram pelo menos um caso entre 2007 e 2014, excluindo-se as localidades sem casos ou silenciosas quanto à presença desses agravos. Foram construídos gráficos de dispersão dos coeficientes de incidência de sífilis (SG e SC) em relação ao percentual de cobertura da ESF. Aplicou-se como teste estatístico a correlação de Spearman $(\rho=$ rho), com o propósito de verificar a relação entre cobertura da ESF e incidência de SC e SG por cada ano em estudo.

Para comparar as diferenças de proporções de cobertura da ESF entre os coeficientes de incidência de SG e SC, no período 2007-2014, utilizou-se o teste exato de Fisher com o método mid-P. ${ }^{10}$ Além disso, foram calculadas as razões de incidências acumuladas, ou seja, as razões das taxas (RT) dos indicadores entre os dois extremos da série.

As análises dos dados foram realizadas pelos programas Microsoft ${ }^{\circledR}$ Excel 2010 e IBM ${ }^{\circledR}$ SPSS Statistics 22.0.
Valores de $\mathrm{p}<0,05$ foram considerados estatisticamente significantes, para todos os testes estatísticos.

0 projeto do estudo foi aprovado pelo Comitê de Ética em Pesquisa da Universidade Federal de Goiás, mediante os Pareceres Consubstanciados $\mathrm{n}^{0} 882.963 / 2014$ e $\mathrm{n}^{0} 1.005 .446 / 2015$, em conformidade com as diretrizes da Resolução do Conselho Nacional de Saúde (CNS) n ${ }^{\circ}$ 466, de 12 de dezembro de 2012.

\section{Resultados}

Entre 2007 e 2014, foram notificados 3.890 casos de SG e 663 casos de SC em todo o estado de Goiás. Do total de casos de SC, 99,0\% (657) foram notificados em menores de 1 ano de idade, 75,2\% deles (499) nascidos de mães que realizaram pré-natal. Somente em 2014, foram notificados 236 casos de SC, representando 35,6\% do total de notificações em todo o período estudado. 0 coeficiente de detecção de SG no estado aumentou em 3,4 vezes no período, indo de 2,8/mil nascidos vivos, em 2007, para 9,5/mil nascidos vivos em 2014 ( $R T=3,4 ; \mathrm{p}<0,001)$. De modo similar, entre 2007 e 2014, houve um aumento de 8,3 vezes no coeficiente de incidência de SC, passando de 0,3 para 2,5 casos por mil nascidos vivos $(\mathrm{RT}=8,3$; $\mathrm{p}<0,001$ ) (Tabela 1).

Em 2014, todas as macrorregiões de saúde de Goiás apresentaram coeficiente de incidência de SC igual ou superior a 0,5 caso por mil nascidos vivos, com destaque para a Sudoeste, que apresentou o maior incremento do coeficiente de incidência entre as macrorregiões do

Tabela 1 - Diferença percentual dos coeficientes de incidência de sífilis congênita, detecção de sífilis em gestante e cobertura da Estratégia Saúde da Família, por Macrorregião de Saúde, Goiás, 2007 e 2014

\begin{tabular}{|c|c|c|c|c|c|c|c|c|c|c|c|c|}
\hline \multirow[t]{2}{*}{ Macrorregião de Saúde } & \multicolumn{4}{|c|}{$\begin{array}{l}\text { Coeficiente de incidência } \\
\text { de sífilis congênita } \\
\text { (por mil nascidos vivos) }\end{array}$} & \multicolumn{4}{|c|}{$\begin{array}{l}\text { Coeficiente de detecção } \\
\text { de sífilis em gestantes } \\
\text { (por mil nascidos vivos) }\end{array}$} & \multicolumn{4}{|c|}{$\begin{array}{c}\text { Cobertura da Estratégia Saúde } \\
\text { da Família } \\
(\%)\end{array}$} \\
\hline & 2007 & 2014 & $\mathbf{R T}^{\mathbf{a}}$ & p-valor ${ }^{b}$ & 2007 & 2014 & $\mathbf{R T}^{\mathbf{a}}$ & p-valor ${ }^{b}$ & 2007 & 2014 & $\mathbf{R T}^{\mathbf{a}}$ & p-valor \\
\hline Centro-0este & 0,2 & 2,9 & 14,5 & $<0,001$ & 2,6 & 9,8 & 3,8 & $<0,001$ & 60,0 & 70,0 & 1,7 & $<0,001$ \\
\hline Nordeste & 0,8 & 0,5 & 0,6 & 0,262 & 3,3 & 5,7 & 1,7 & $<0,001$ & 52,0 & 66,0 & 1,3 & $<0,001$ \\
\hline Centro-Norte & 0,3 & 2,4 & 8,0 & $<0,001$ & 3,7 & 8,3 & 2,2 & $<0,001$ & 71,0 & 82,0 & 1,5 & $<0,001$ \\
\hline Sudoeste & 0,1 & 2,6 & 26,0 & $<0,001$ & 4,1 & 11,4 & 2,8 & $<0,001$ & 58,0 & 59,0 & 1,0 & $<0,001$ \\
\hline Centro-Sudeste & 0,2 & 3,2 & 16,0 & $<0,001$ & 1,4 & 11,1 & 7,9 & $<0,001$ & 61,0 & 70,0 & 1,1 & $<0,001$ \\
\hline Goiás & 0,3 & 2,5 & 8,3 & $<0,001$ & 2,8 & 9,5 & 3,4 & $<0,001$ & 55,9 & 64,6 & 1,5 & $<0,001$ \\
\hline
\end{tabular}

a) RT: razão de taxas.

b) Teste exato de Fisher com método mid-P. 
estado ( $\mathrm{RT}=26,0 ; \mathrm{p}<0,001)$. Apenas a macrorregião Nordeste apresentou queda no indicador de SC entre 2007 e 2014, embora sem significância estatística ( $\mathrm{RT}=0,6 ; \mathrm{p}=0,262)$. No mesmo período (2007-2014), houve um aumento no coeficiente de detecção de $S G$ em todas as macrorregiões de saúde $(\mathrm{p}<0,001)$ (Tabela 1$)$.

Em 2007, 51 municípios notificaram casos de SG, chegando a 127 em 2014: uma variação positiva de 149,0\% durante o período. Em 2007, 15 municípios notificaram pelo menos um caso de SC; em 2014, esse número aumentou para 54 municípios (Figuras 1 e 2).

Observou-se correlação positiva entre 0 aumento percentual da cobertura da ESF e o aumento no coeficiente de detecção de SG em quase todos os anos $(\mathrm{p}<0,05)$; a exceção coube a $2012(\rho=0,106$; $\mathrm{p}=0,320)$ (Figura 1). Essa correlação positiva também foi observada em relação à incidência de SC na maioria dos anos estudados $(\mathrm{p}<0,05)$; aqui, a exceção coube a $2008(\rho=0,477 ; p=0,117), 2012(\rho=0,463$; $p=0,053)$ e $2014(\rho=0,241 ; p=0,079)$, anos em que não se alcançou significância estatística (Figura 2).

Ao se comparar os anos extremos da série, 2007 e 2014, observou-se aumento significativo do coeficiente de incidência de SC entre os municípios com cobertura da ESF $\leq 25 \%(\mathrm{RT}=5,8 ; \mathrm{p}<0,001),>25 \%$ e $<50 \%(\mathrm{RT}=7,5 ; \mathrm{p}<0,001)$ e $\geq 50 \%$ e $<75 \%(\mathrm{RT}=1,6$; $\mathrm{p}$-valor $<0,001)$. Entretanto, no estrato de maior cobertura da $\operatorname{ESF}(\geq 75 \%)$, não se encontrou diferença estatística no coeficiente de incidência de SC entre 2007 e 2014 (RT=0,9; p=0,602) (Tabela 2).

\section{Discussão}

0 número de municípios notificantes e a incidência de SG e SC no estado de Goiás aumentaram entre 2007 e 2014. 0 percentual de cobertura da ESF apresentou correlação positiva com o número de casos de SG e de SC, na maioria dos anos estudados. Destaca-se que o aumento do número de casos de SC entre $2007 \mathrm{e}$ 2014 foi mais evidente nos municípios com cobertura da ESF inferior a $75 \%$.

A partir do delineamento do presente estudo, não é possível comprovar relação causal entre a cobertura da ESF nos municípios e o número de casos de SG e SC. A cobertura da ESF foi utilizada como medida indireta de acesso ao pré-natal; contudo, não se trata de um único indicador de acesso a esse serviço. ${ }^{11,12} \mathrm{~A}$ utilização de dados secundários, oriundos de notificação passiva de casos de sífilis, pode representar apenas uma parcela dos casos existentes. Ao mesmo tempo, acredita-se que a possível presença de subnotificação não mudaria substancialmente a direção dos resultados encontrados no presente estudo, pois houve crescimento dos coeficientes ao longo do período. Existe também a possibilidade de superestimar o número de casos, em decorrência das dificuldades na investigação desses casos para confirmação do diagnóstico de SC, uma vez que se mantiveram os mesmos critérios de notificação durante o período analisado.

No presente estudo, investigou-se a relação entre incidência de sífilis (SC e SG) e cobertura da ESF, independentemente do tempo de atuação e experiência das equipes. Ademais, não foi feito nenhum tipo de ajuste em relação ao perfil sociodemográfico dos municípios notificantes.

O crescimento do número de casos de SG e SC em Goiás e no Brasil, sobretudo a partir de 2012, pode sugerir uma ampliação das ações de vigilância, além de apontar para um aumento real do número de casos advindos da expansão das infecções sexualmente transmissíveis (IST), crescimento este observado em diferentes regiões do mundo. ${ }^{13,14}$

A maior ocorrência da sífilis adquirida nos estados brasileiros, nos últimos anos, sugere um crescimento de práticas sexuais desprotegidas, impactando no crescimento dos casos de SG e repercutindo na dificuldade de interrupção da cadeia de transmissão da sífilis, principalmente pela reinfecção da gestante via parcerias sexuais não tratadas. . $^{14,15}$

Em 2015, foi lançado o 'Caderno de Boas Práticas: 0 uso da penicilina na atenção básica para a prevenção da sífilis congênita no Brasil', cuja ênfase se encontrava na necessidade de início do tratamento da gestante, sem hesitação, por parte da equipe de saúde. Esta recomendação se justificava pelos raros índices de reações adversas e pela existência de protocolo de atendimento específico para situações de urgência na Atenção Básica. ${ }^{15}$ Outra prática importante foi a priorização da gestante quanto ao uso da penicilina em caso de desabastecimento do fármaco. ${ }^{16}$

Não obstante a existência dessas e de outras estratégias para garantia do uso da penicilina pela gestante na Atenção Básica, especialmente a partir de 2015, o aumento dos casos em 2014, último ano da série estudada, pode estar relacionado a fatores como: a falta do fármaco no mercado; a descentralização na distribuição dos testes rápidos de sífilis na Atenção Básica, aumentando o acesso à triagem da infecção; 


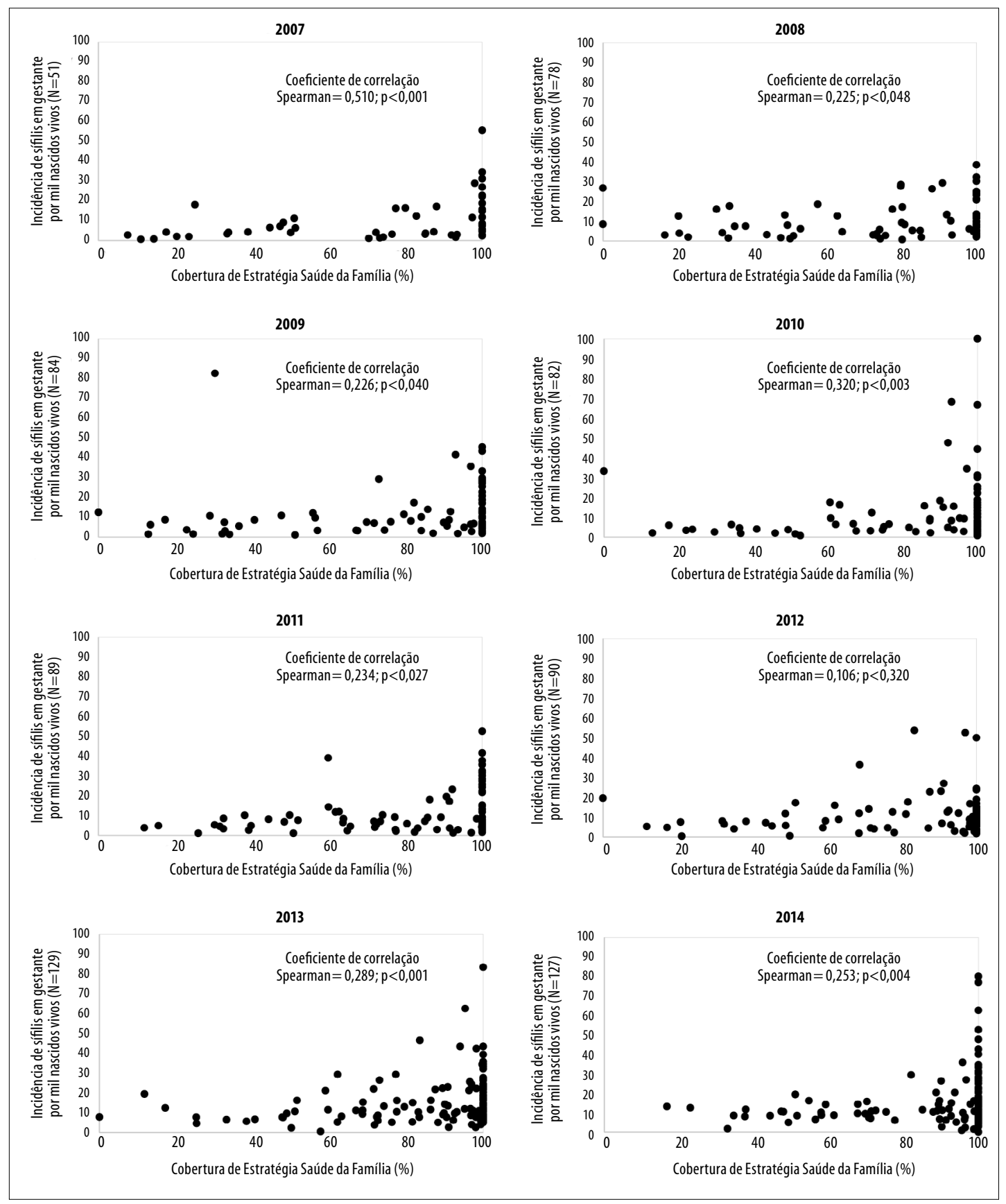

\section{Figura 1 - Correlação entre a cobertura da Estratégia Saúde da Família e o coeficiente de detecção de sífilis em gestantes, Goiás, 2007-2014}

ou ainda o tratamento tardio e inadequado da infecção, com perdas de seguimento do paciente na rede de atendimento. ${ }^{5,17,18}$

A correlação positiva entre o coeficiente de detecção de SG e a cobertura da ESF, observada neste estudo, reforça a hipótese de expansão da cobertura de testagem para sífilis e da notificação dos casos de gestantes infectadas no âmbito da ESF, ações estas que contribuem para o fortalecimento dos serviços de pré-natal, sobretudo após a implantação da Rede Cegonha, em 2011, e do Programa de Qualificação das Ações de Vigilância em Saúde, em 2013. ${ }^{8,19}$ 


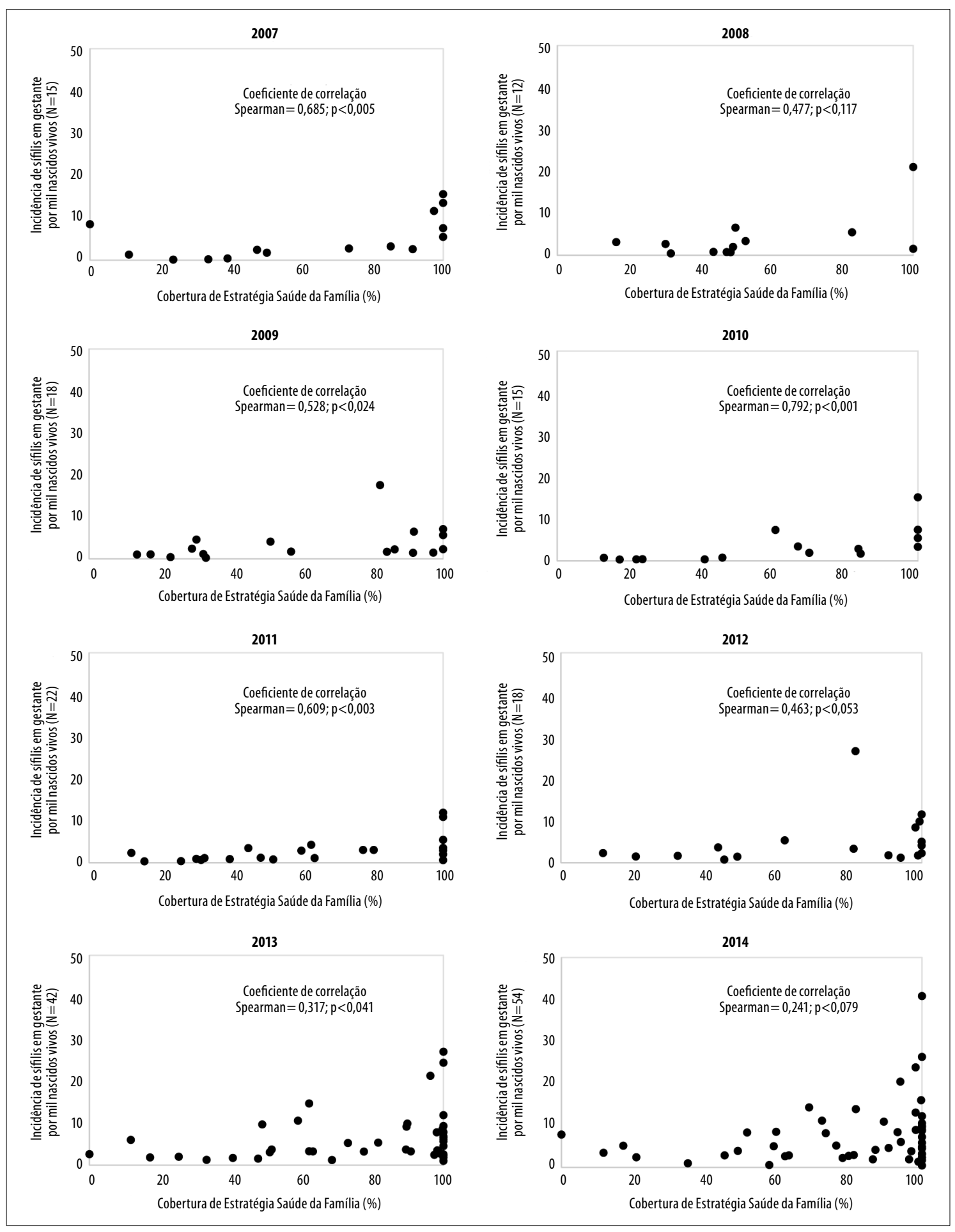

Figura 2 - Correlação entre a cobertura da Estratégia Saúde da Família e o coeficiente de incidência de sífilis congênita, Goiás, 2007-2014 
Tabela 2 - Variação dos coeficientes de incidência de sífilis congênita por estratos de cobertura da Estratégia Saúde da Família, Goiás, 2007 e 2014

\begin{tabular}{|c|c|c|c|c|c|c|}
\hline \multirow{2}{*}{$\begin{array}{l}\text { Cobertura da Estratégia } \\
\text { Saúde da Família (\%) }\end{array}$} & \multicolumn{2}{|c|}{ Municípios } & \multicolumn{2}{|c|}{$\begin{array}{l}\text { Coeficiente de incidência de sífilis congênita } \\
\text { (por mil nascidos vivos) }\end{array}$} & \multirow[t]{2}{*}{$\mathbf{R} \mathbf{T}^{\mathbf{a}}$} & \multirow{2}{*}{$\frac{p \text {-valor }}{2007}$} \\
\hline & $\begin{array}{c}2007 \\
(n=15)\end{array}$ & $\begin{array}{c}2014 \\
(n=54)\end{array}$ & 2007 & 2014 & & \\
\hline$\leq 25$ & 3 & 4 & 0,6 & 3,5 & 5,8 & $<0,001$ \\
\hline$>25$ e $<50$ & 3 & 3 & 0,4 & 3,0 & 7,5 & $<0,001$ \\
\hline$\geq 50 \mathrm{e}<75$ & 2 & 9 & 2,8 & 4,5 & 1,6 & $<0,001$ \\
\hline$\geq 75$ & 7 & 38 & 5,7 & 5,1 & 0,9 & 0,602 \\
\hline
\end{tabular}

a) RT: razão de taxas.

b) Teste exato de Fisher com método mid-P.

A SC é considerada evento sentinela da qualidade da assistência pré-natal. Ações de saúde eficazes são capazes de evitá-la. ${ }^{20}$ Municípios com percentual de cobertura da ESF inferiores a 75\% apresentaram maior crescimento do número de casos de SC ao longo do período estudado, se comparados àqueles com coberturas superiores. Neste contexto, além da necessidade de ampliação do acesso, é importante investigar a capacidade resolutiva da rede de serviços de atenção primária à saúde no estado, com vistas à proposição de melhorias no acesso ao diagnóstico e, principalmente, à intervenção adequada e oportuna, aspectos também apontados como fragilidades na interrupção da cadeia de transmissão. Assim concluíram estudos realizados no Ceará e em São Paulo. ${ }^{21,22}$

A expansão da cobertura da ESF nas macrorregiões de saúde de Goiás acompanhou a tendência nacional. Segundo o DAB/Ministério da Saúde, no Brasil como um todo, ao longo de 15 anos, ampliou-se em mais de quatro vezes a população coberta pela Saúde da Família, passando de 14,1\% em 2000 para 59,7\% em 2014. ${ }^{23}$ No estado de Goiás, entre 2007 e 2014, foram implantadas 240 equipes de saúde da família, passando de 55,9 para 64,6\% de cobertura da ESF. ${ }^{23}$

Não houve diminuição significativa de SC nos municípios com maior cobertura da $\mathrm{ESF}(\geq 75 \%)$ no período em estudo, o que leva à necessidade de se investigar outros fatores intervenientes no enfrentamento dessa infecção congênita, como as condições sociodemográficas e de assistência à saúde nesses municípios. Estudo de série temporal, realizado no país entre 2003 e 2008, mostrou redução mais intensa de $\mathrm{SC}$ nos municípios com maiores estratos de cobertura da ESF; contudo, a correlação negativa entre incidência de SC e cobertura da ESF não se manteve após ajuste por potenciais variáveis de confusão, sugerindo que a redução da SC não seria atribuível à ESF e sim à cobertura de pré-natal e às características demográficas dos municípios. ${ }^{24}$

Outro aspecto importante a salientar, e um desafio à prevenção da transmissão vertical da sífilis, é o treinamento adequado dos profissionais de saúde, sobretudo daqueles lotados nos serviços de pré-natal. ${ }^{25}$ Estudo realizado no Brasil sobre internações por condições sensíveis à atenção primária mostrou que a ESF tem contribuído com avanços no enfrentamento de alguns agravos; porém, a mesma investigação destacou a necessidade de capacitação das equipes e um melhor direcionamento das ações. ${ }^{26}$

Mais uma pesquisa nacional, conduzida entre 2011 e 2012, mostrou que, apesar da elevada cobertura de pré-natal, tão somente um quinto das mulheres atendidas recebeu cuidados adequados aos procedimentos mínimos preconizados pelo Ministério da Saúde. De acordo com a referida pesquisa, menos de $50 \%$ das gestantes realizaram duas sorologias para sífilis durante a gestação, conforme recomendado. Tais fatores reduzem a possibilidade de intervenção adequada e contribuem para 0 aumento dos casos de transmissão vertical, como revela o presente estudo. ${ }^{19,27}$

As dificuldades encontradas na luta contra a sífilis são inúmeras. Com o objetivo de melhor elucidar os principais entraves no controle da transmissão vertical do agravo, no contexto das diferentes realidades brasileiras, o Ministério da Saúde incentiva a criação de comitês de investigação dos casos de SC. Ademais, a instituição lançou, no final de 2014, o 'Protocolo de Investigação de Transmissão Vertical' no âmbito da vigilância, prevenção e controle das IST, do HIV/aids e das hepatites virais, contemplando casos de SC precoce. A publicação 
constitui uma ferramenta norteadora para a investigação das vulnerabilidades individuais, dos serviços assistenciais e daquelas dificuldades relacionadas à gestão, que impactam diretamente no aumento dos casos. ${ }^{28}$

A ESF é um espaço privilegiado de intervenção territorial. Todavia, faz-se mister aprofundar a discussão da problemática da SG e da SC na vertente de política pública intersetorial, de forma a se integrar a ESF com outros pontos das redes de atenção à saúde, e destes com outros setores sociais. ${ }^{6}$ Outrossim, deve-se incentivar a criação de espaços de participação social para a construção conjunta de ações com poder de impacto efetivo sobre a realidade dessa infecção.

A incidência de SG e SC aumentou ao longo do período, e apresentou correlação positiva com 0 aumento da ESF na maioria dos anos estudados. Observa-se expansão da epidemia e perdas de oportunidades de prevenção da transmissão vertical da doença, inclusive

\section{Referências}

1. Newman L, Kamb M, Hawkes S, Gomez G, Say L, Seuc A, et al. Global estimates of syphilis in pregnancy and associated adverse outcomes: analysis of multinational antenatal surveillance data. PLoS Med. 2013;10(2):e1001396. doi: 10.1371/journal. pmed.1001396.

2. World Health Organization. Global guidance on criteria and processes for validation: elimination of mother-to-child transmission (EMTCT) of HIV and Syphilis [Internet]. Geneve: World Health Organization; 2014 [cited 2018 Jul 3]. 32 p. Available from: http://apps.who.int/iris/ bitstream/handle/10665/112858/9789241505888_eng. pdf?sequence $=1$

3. Hawkes S J, Gomez, GB, Broutet N. Early antenatal care: does it make a difference to outcomes of pregnancy associated with syphilis? A systematic review and metaanalysis. PLoS One. 2013;8(2):e56713. doi: 10.1371/ journal.pone.0056713.

4. Mesquita KO, Lima GK, Filgueira AA, Flôr SMC, Freitas CASL, Linhares MSC, et al. Análise dos casos de sífilis congênita em Sobral, Ceará: contribuições para assistência pré-natal. DST-J Bras Doenças Sex Transm. 2012;24(1):20-7. doi: 10.5533/2177-8264-201224107.

5. Cardoso ARP, Araújo MAL, Cavalcante MS, Frota MA, Melo SP. Análise dos casos de sífilis gestacional e congênita nos anos de 2008 a 2010 em Fortaleza, Ceará, Brasil. Ciênc Saúde Coletiva. 2018 fev;23(2):563-74. doi: 10.1590/141381232018232.01772016 . em municípios com altas coberturas da Estratégia Saúde da Família, indicando a necessidade de fortalecimento da Atenção Básica, sobretudo nos aspectos relacionados à qualidade do pré-natal e da investigação dos fatores envolvidos na transmissão vertical da sífilis, visando a intervenções mais assertivas.

\section{Contribuição das autoras}

Nunes PS e Turchi MD participaram da concepção e delineamento do estudo, análise e interpretação dos dados e redação do manuscrito. Zara ALSA, Rocha DFNC, Marinho TA e Mandacarú PMP participaram da análise e interpretação dos dados e de revisão crítica relevante do conteúdo intelectual do manuscrito. Todas as autoras aprovaram a versão final e declaram ser responsáveis por todos os aspectos do trabalho, incluindo a garantia de sua precisão e integridade.

6. Arantes LJ, Shimizu HE, Merchán-Hamann E. Contribuições e desafios da estratégia saúde da família na atenção primária à saúde no Brasil: revisão da literatura. Ciênc Saúde Coletiva. 2016 maio;21(5):1499-510. doi: 10.1590/1413-81232015215.19602015.

7. Gomez GB, Kamb ML, Newman LM, Mark J, Broutet $\mathrm{N}$, Hawkes SJ. Untreated maternal syphilis and adverse outcomes of pregnancy: a systematic review and meta-analysis. Bull World Health Organ. 2013 Mar;91(3):217-26. doi: 10.2471/BLT.12.107623.

8. Brasil. Ministério da Saúde. Portaria MS/GM n ${ }^{\circ}$ 1.459, de 24 de julho de 2011. Institui, no âmbito do Sistema Único de Saúde - SUS - a Rede Cegonha. Diário Oficial da República Federativa do Brasil, Brasília (DF), 2011 jul 11; Seção I, p. 109.

9. Instituto Brasileiro de Geografia e Estatística. Estados@ Goiás 2015 [Internet]. 2018 [citado 2018 out 5]. Disponível em: http://www.ibge.gov.br/ estadosat/perfil.php?lang $=\&$ sigla $=$ go

10. Martin DO, Austin H. Exact estimates for a rate ratio. Epidemiology. 1996 Jan;7(1):29-33.

11. Ministério da Saúde (BR). Secretaria de Atenção à Saúde. Departamento de Ações Programáticas Estratégicas. Gestação de alto risco: manual técnico [Internet]. 5.ed. Brasília: Ministério da Saúde; 2012 [citado 2018 out 5]. 302 p. Disponível em: http://bvsms.saude.gov.br/bvs/publicacoes/manual_ tecnico_gestacao_alto_risco.pdf 
12. Ministério da Saúde (BR). Programa nacional de melhoria do acesso e da qualidade da atenção básica (PMAQ): ficha de qualificação de indicadores [Internet]. Brasília: Ministério da Saúde; 2012 [citado 2018 out 5]. 80p. Disponível em: http://189.28.128.100/dab/docs/sistemas/Pmaq/ manual_instrutivo_pmaq_site_anexo.pdf

13. Kenyon C, Buyze J, Colebunders R. Classification of incidence and prevalence of certain sexually transmitted infections by world regions. Int J Infect Dis. 2014 Jan;18:73-80. doi: 10.1016/j. ijid.2013.09.014.

14. Ministério da Saúde (BR). Secretaria de Vigilância em Saúde. Sífilis 2017. Bol Epidemiol. 2017 nov;48(36):1-41.

15. Ministério da Saúde (BR). Secretaria de Vigilancia em Saúde. Departamento de DST, Aids e Hepatites Virais. Caderno de boas práticas: o uso da penicilina na atenção básica para a prevenção da sífilis congênita no Brasil [Internet]. Brasília: Ministério da Saúde; 2015 [citado 2018 out 5]. 96 p. Disponível em: http://bvsms.saude.gov.br/bvs/publicacoes/penicilina_ para_prevencao_sifilis_congenita\%20_brasil.pdf

16. Brasil. Ministério da Saúde. Secretaria de Vigilância em Saúde. Secretaria de Ciência, Tecnologia e Insumos Estratégicos. Nota informativa conjunta $\mathrm{n}^{\circ}$ 109/2015 GAB/SVS/MS, GAB/SCTIE/MS, de 28 outubro de 2015 . Orienta a respeito da priorização $\mathrm{da}$ penicilina $\mathrm{G}$ benzatina para sífilis em gestantes $\mathrm{e}$ penicilina cristalina para sífilis congênita no país e alternativas para o tratamento da sífilis [Internet]. Brasília: Ministério da Saúde, 2015 [citado 2018 out 5]. 3 p. Disponível em: http://www.aids.gov. br/pt-br/legislacao/nota-informativa-conjunta-no109105gabsvsms-gabsctiems

17. Pan American Health Organization. Elimination of mother-to-child transmission of HIV and syphilis in the Americas. Update 2016 [Internet]. Washington, D.C.: Pan American Health Organization; 2016 [cited 2018 Oct 5]. 62 p. Available: http://iris.paho.org/xmlui/bitstream/ha ndle/123456789/34072/9789275119556-eng. pdf?sequence $=4$ \&isAllowed $=\mathrm{y}$

18. Taylor MM, Nurse-Findlay S, Zhang X, Hedman L, Kamb ML, Broutet N, et al. Estimating benzathine penicillin need for the treatment of pregnant women diagnosed with syphilis during antenatal care in high-morbidity countries. PLoS One. 2016 Jul;11(7):e0159483. doi: 10.1371/journal.pone.0159483.

19. Ministério da Saúde (BR). Secretaria de Vigilância em Saúde. Programa de qualificação das ações de vigilância em saúde (PQA-VS): ficha de qualificação dos indicadores [Internet]. Brasília: Ministério da Saúde; 2013 [citado 2018 out 5] 18 p. Disponível em: http://www.saude.sp.gov.br/resources/ccd/homepage/ acesso-rapido/gpa/caderno_indicadores_do_pqavs.pdf

20. Domingues RMSM, Saracen V, Hartz ZMA, Leal MC. Sífilis congênita: evento sentinela da qualidade da assistência pré-natal. Rev Saúde Pública. 2013 fev;41(1):147-57. doi: 10.1590/S0034891020130001.00019 .

21. Costa CC, Freitas LV, Sousa DMN, Oliveira LL, Chagas ACMA, Lopes MVO et al. Sífilis congênita no Ceará: análise epidemiológica de uma década. Rev Esc Enferm USP. 2013 fev;47(1):152-9. doi: 10.1590/ S0080-623420130001.00019.

22. Donalísio MR, Freire JB, Mendes ET. Investigação da sífilis congênita na microrregião de Sumaré, Estado de São Paulo, Brasil - desvelando a fragilidade do cuidado à mulher gestante e ao recém-nascido. Epidemiol Serv Saúde. 2007 jul-set;16(3):165-73. doi: 10.5123/S1679-49742007000300003.

23. Ministério da Saúde (BR). Departamento de Atenção Básica. Histórico de cobertura saúde da família [Internet]. 2015 [citado 2018 out 5]. Disponível em: http://dab. saude.gov.br/portaldab/historico_cobertura_sf.php

24. Araújo CL, Shimizu HE, Sousa AIA, Hamann EM. Incidência da sífilis congênita no Brasil e sua relação com a estratégia saúde da família. Rev Saúde Pública. 2012 jun;46(3):47986. doi: 10.1590/S0034-8910201200300010.

25. Kiarie J, Mishra CK, Temmerman M, Newman L. Accelerating the dual elimination of mother-to-child transmission of syphilis and HIV: why now? Int J Gynaecol Obstet. 2015 Jun;130(Suppl 1):S1-3. doi: 10.1016/j.ijgo.2015.05.002.

26. Ceccon RF, Meneghel SN, Viecili PR. Hospitalization due to conditions sensitive to primary care and expansion of the Family Health Program in Brazil: an ecological study. Rev Bras Epidemiol. 2014 Dec;17(4):968-77.

27. Domingues RM, Dias MA, Leal MC, Gama SG, ThemeFilha MM, Torres JA, et al. Adequacy of prenatal care according to maternal characteristics in Brazil. Rev Panam Salud Publica. 2015 Mar;37(3):140-7.

28. Ministério da Saúde (BR). Protocolo de infestigação de transmissão vertical [Internet]. Brasília: Ministério da Saúde; 2014 [citado 2018 out 5]. 83 p. Disponível em: http://www.aids.gov.br/pt-br/pub/2014/protocolode-investigacao-de-transmissao-vertical 


\begin{abstract}
Objective: to analyze the incidence of syphilis in pregnant women (SPW) and congenital syphilis (CS) and the correlation of these indicators with Family Health Strategy (FHS) coverage in Goiás, Brazil, from 2007 to 2014. Methods: this was an ecological study using Notifiable Diseases Information System (SINAN) data and data from the Health Ministry's Department of Primary Care; Spearman's correlation was used to evaluate the relationship between FHS coverage and syphilis incidence. Results: SPW incidence increased from 2.8 to 9.5/1,000 live births, while CS incidence increased from 0.3 to 2.5/1,000 live births $(p<0.05)$ in the period 2007-2014; there was a significant increase in CS cases in municipalities with percentage FHS coverage below $75 \%(p<0.001)$. Conclusion: the concomitant increase in SPW and CS incidence suggests failures in the prevention of vertical transmission of syphilis, especially in regions with lower FHS coverage.
\end{abstract}

Keywords: Syphilis; Syphilis, Congenital; Pregnant Women; Family Health Strategy; Ecological Studies.

\section{Resumen}

Objetivo: analizar la incidencia de sífilis en gestante (SG) y sífilis congénita (SC) y la correlación de esos indicadores con la cobertura de la Estrategia Salud de la Familia (ESF) en Goiás, Brasil, de 2007 a 2014. Métodos: estudio ecológico utilizando datos del Sistema de Información de Agravamientos de Notificación (Sinan) y del Departamento de Atención Básica (DAB)/Ministerio de la Salud; se utilizó la correlación de Spearman para evaluar la relación entre la cobertura de la ESF y la incidencia de sifilis. Resultados: la incidencia de SG pasó de 2,8 a 9,5/1.000 nacidos vivos, y la de SC, de 0,3 a 2,5/1.000 nacidos vivos $(p<0,05)$ en el periodo 20072014; bubo un aumento significativo de casos de SC en los municipios que presentaron un porcentaje de cobertura de la ESF inferior al 75\% $(p<0,001)$. Conclusión: el aumento concomitante de la incidencia de $S G$ y de SC sugiere fallas en la prevención de la transmisión vertical de la sifilis, sobre todo en las regiones con menor porcentaje de cobertura de la ESF.

Palabras-clave: Sífilis; Sifilis Congénita; Mujeres Embarazadas; Estrategia de Salud Familiar; Estudios Ecológicos.

Recebido em 27/05/2018

Aprovado em 25/09/2018 